\title{
Artifactual measurement of low serum HDL-cholesterol due to paraproteinemia
}

\author{
Edwin O. F. van Gorselen - Theo Diekman • \\ Jan Hessels • Patrick M. J. Verhorst • \\ Clemens von Birgelen
}

Received: 7 January 2010/Accepted: 17 June 2010/Published online: 1 July 2010

(C) The Author(s) 2010. This article is published with open access at Springerlink.com

\section{Sirs:}

High levels of serum low density lipoprotein cholesterol (LDL-C) and low levels of high density lipoprotein cholesterol (HDL-C) are well-known risk factors for premature atherosclerotic vascular disease [1,2]. They are targets for primary and secondary prevention. Interpreting lipid profiles is part of the daily routine for a cardiologist. The most common cause of low HDL-C in western society is metabolic syndrome. More rare are primary lipid disorders (e.g., Tangier syndrome due to an ABCA transporter deficiency or deficiency of apolipoprotein A1) and secondary causes like (ab)use of androgens (Table 1). Extremely low serum HDL levels are associated with an increased risk of death, sepsis and malignancy [3]. A rare but important cause is interference in the biochemical assay by paraproteins, yielding an artifactually low HDL-C measurement result. We present the case of a patient who had his lipid profile

E. O. F. van Gorselen $(\varangle) \cdot$ P. M. J. Verhorst · C. von Birgelen Department of Cardiology, Medisch Spectrum Twente,

Thoraxcentrum Twente, Haaksbergerstraat 55,

7513ER Enschede, The Netherlands

e-mail: e.vangorselen@mst.nl

T. Diekman

Department of Internal Medicine, Deventer Ziekenhuis, Deventer, The Netherlands

J. Hessels

Department of Clinical Chemistry, Deventer Ziekenhuis, Deventer, The Netherlands

C. von Birgelen

MIRA-Institute for Biomedical Technology and Technical Medicine, University of Twente,

Enschede, The Netherlands repeatedly tested over the course of 4 years and had progressive decline in HDL-C measurements.

A 74-year-old man presented with fever, cold chills, vomiting, diarrhea and shortness of breath. He did not use any chronic medication. Upon physical examination, he was dyspnoeic with a respiratory rate of 30 per min, an oxygen saturation of $87 \%$ at ambient air, a temperature of $39^{\circ} \mathrm{C}$, and a blood pressure of $170 / 120 \mathrm{mmHg}$ with a heart rate of $135 \mathrm{bpm}$. On auscultation of the heart, there was a regular tachycardia without murmurs. Crackles and rhonchi were present at the base of the right lung. There was no lymphadenopathy or hepatosplenomegaly. The chest X-ray revealed a large infiltration in the right lung. Respiratory insufficiency and evolving sepsis required admittance to the ICU with mechanical ventilation and vasopressive medication. Initial antibiotic treatment consisted of moxifloxacin with a switch to penicillin $\mathrm{G}$ after blood cultures grew Streptococcus pneumonia. The patient developed renal insufficiency due to an acute tubulus necrosis, requiring temporary continuous veno-venous haemofiltration. After 1 week, he could be transferred to the internal medicine ward. Recovery and mobilization were relatively slow in part due to a parapneumonic effusion. When the patient was discharged to a revalidation center (37 days after admission), vital parameters and renal function were normalized.

Three months after his first hospitalization he was re-admitted with pneumonia. Re-evaluation of the laboratory reports of the first hospital episode revealed at presentation an ESR of $132 \mathrm{~mm} / \mathrm{h}$ and normochromic anemia with a hemoglobin value of $6.0 \mathrm{mmol} / \mathrm{l}$. Three months before admission, a low HDL-C of $0.1 \mathrm{mmol} / \mathrm{l}$ had been measured. Because of the recurrent nature of the infection, an immune compromising condition was suspected. Multiple myeloma with paraproteinemia was detected with a 
Table 1 Differential diagnosis: Causes of low HDL-C values [adapted from 4-7]

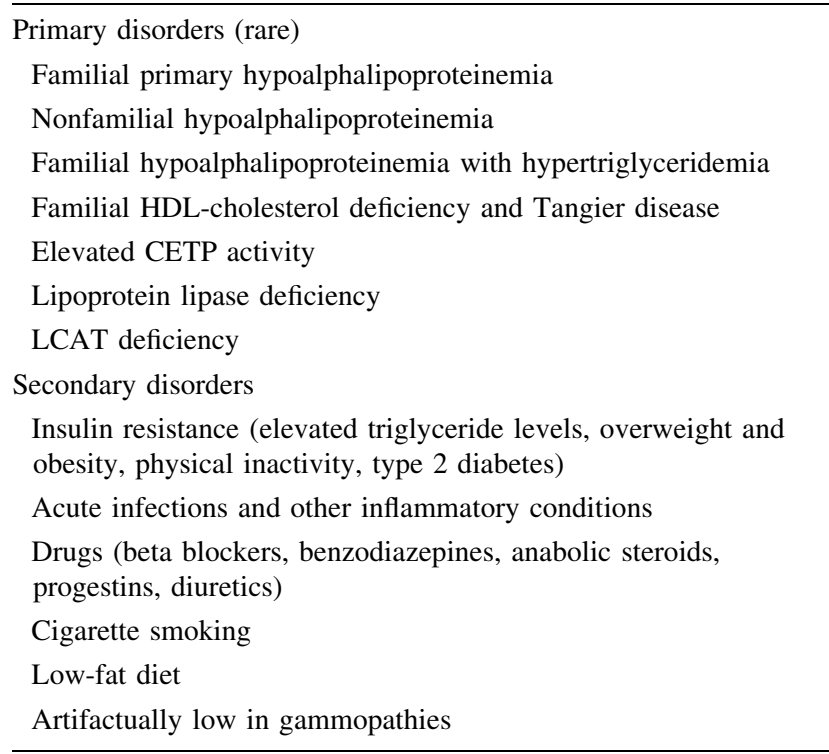

monoclonal IgG- $\lambda$ of $41.7 \mathrm{~g} / \mathrm{l}$ (Fig. 1) and a plasma beta-2 microglobuline of $7.2 \mathrm{mg} / \mathrm{l}$; plasma calcium was normal. The skeletal survey showed no signs of lytic activity. A peripheral blood smear showed rouleaux formation (pseudo agglutination) of erythrocytes.

The bone marrow biopsy was hypercellullar, mainly consisting of plasma cells. There was a monoclonal plasma cell proliferation $(47.2 \%)$, consistent with multiple myeloma. Immunophenotyping and in situ hybridization showed a monoclonality for IgG- $\lambda$. Flow cytometry showed CD56, CD38, CD138 positivity and CD19 and CD45 negativity (Fig. 2). According to the criteria of the International Staging System for multiple myeloma, there was a stage 3 multiple myeloma [8]. Therapy was started with melfalan, prednisolon, thalidomide and later bortezomib with dexamethason with good hematologic response: $\mathrm{IgG}-\lambda$ dropped to $3 \mathrm{~g} / \mathrm{l}$. After correction of the paraproteinemia with this therapy HDL-C restored to a level of $1.0 \mathrm{mmol} / \mathrm{l}$ (Table 2). Because of the prothrombogenic side effects of thalidomide, acetylsalicylic acid was added for prophylaxis. Besides a short hospital admission due to leucopenic fever after 8 months, the patient was symptom-free at 1 -year clinical follow-up.

HDL-C measurements were initially performed on a Hitachi 917 analyzer (Roche Diagnostics, Switzerland) with HDL-C reagent (HDL-C plus second generation, Roche Diagnostics, Switzerland). A reanalysis of the serum sample with high paraproteins with a conventional precipitation method (sodium phosphotungstate- $\mathrm{MgCl}_{2}$ ) yielded a normal value for HDL-C of $1.2 \mathrm{mmol} / \mathrm{l}$.

The case presented contains several learning points. First, the laboratory charts disclose a steady decline of HDL-C values over a period of 4 years, reflecting the development of increasing amounts of paraproteins which interfered in the HDL-C assay.

Secondly, interference of paraproteins with HDL-C measurement can occur in various automated analyzers [10-14]. Paraproteins may have unusual specificity for various antigens, including actin, double-stranded DNA, thyroglobulin, insulin, and apolipoprotein [15]. Furthermore, paraproteins may also interfere with the measurement of HDL-C in homogeneous automated assays [10-14]. Interference is most probably due to the formation of a precipitate with the first reagent [12]. The first reagents used in this assay consists of dextransulphate, $\mathrm{MgSO}_{4}$ and $\alpha$-cyclodextrin. These reagents are used to block the low density lipoproteins (i.e. to make them inaccessible for the later added cholesteroloxidase). In this way, only cholesterol of HDL particles can be converted in cholestenone and $\mathrm{H}_{2} \mathrm{O}_{2}$. Using peroxidase, the formed peroxide can finally be converted into color, which can then be

Fig. 1 Densitometric scan of protein electrophoresis (left) with five protein fractions from left to right: albumin, alpha 1 , alpha 2, beta and gamma. Electrophoresis (ELP) and immunofixation of serum (right) with anti-IgG $(G)$, anti $\operatorname{IgA}(A)$, anti $\operatorname{IgM}(M)$, anti-kappa $(K)$ and anti lambda $(L)$

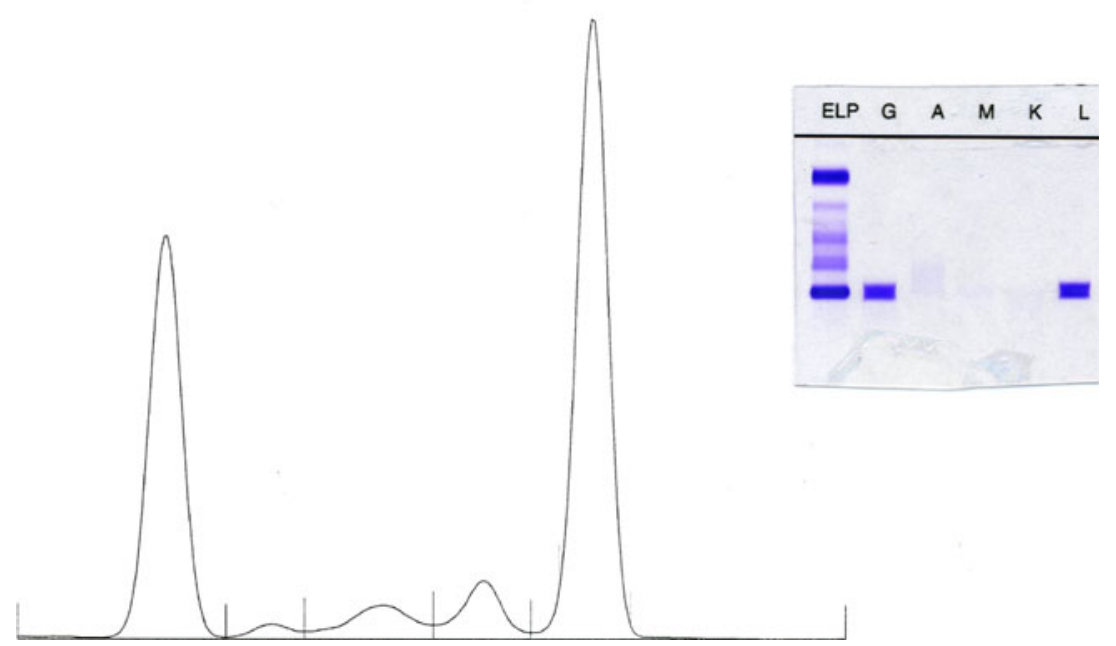




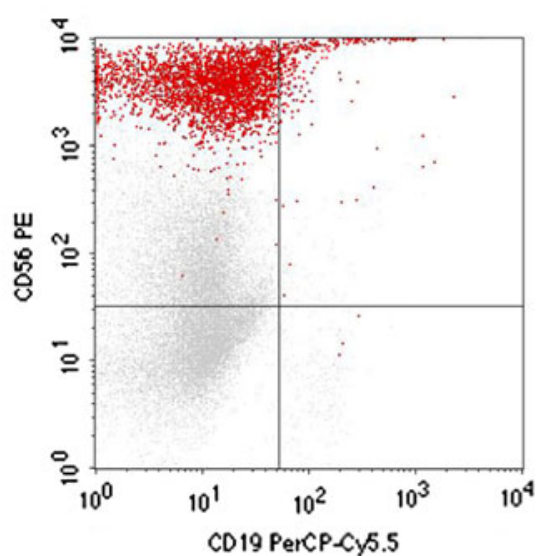

CD 56+/CD19-

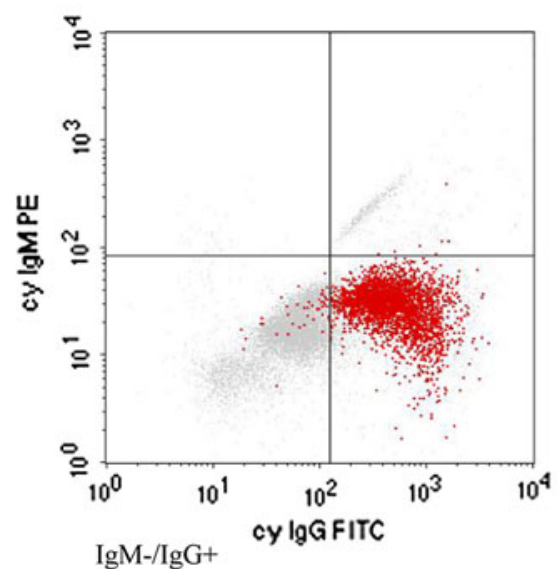

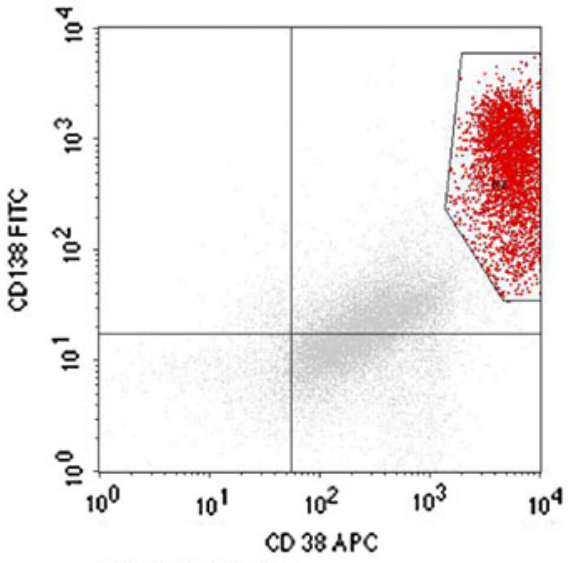

CD $138+/ \mathrm{CD} 38+$

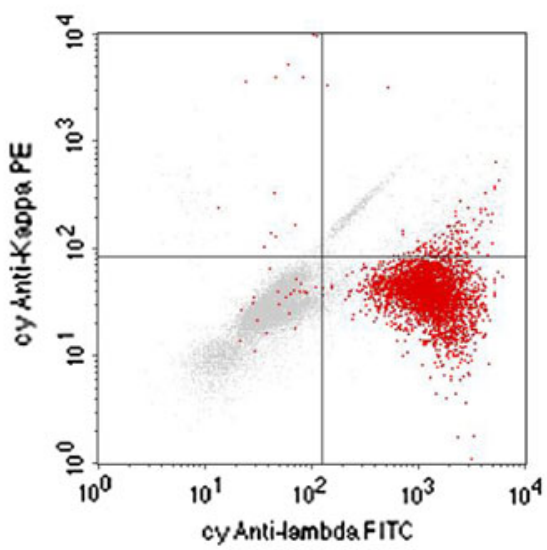

anti-kappa-/anti-lambda +

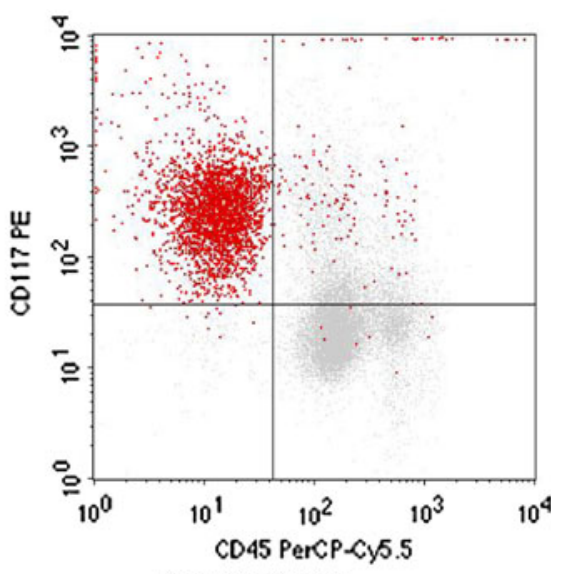

CD $117+/$ CD $45-$

Fig. 2 Immunophenotyping of bone marrow aspirate by flow cytometry. FACS (fluorescence activated cell sorting) plots of CD 56-CD 19, CD 138-CD 38, CD 117-CD 45 were consistent with the

diagnosis multiple myeloma $\operatorname{IgG}-\lambda$. Myeloma cells are typically CD56, CD38, CD138-positive and CD19 and CD45-negative [9]

Table 2 Chart with results of laboratory tests

\begin{tabular}{|c|c|c|c|c|c|c|}
\hline & \multirow[t]{2}{*}{ Reference values } & \multicolumn{5}{|c|}{ Years before and after presentation } \\
\hline & & -4 & -3 & -1 & 0 & 1 \\
\hline Cholesterol & $3.5-5.0 \mathrm{mmol} / \mathrm{l}$ & 4.9 & 4.1 & 3.8 & 3.6 & 5.5 \\
\hline HDL-C & $0.9-1.7 \mathrm{mmol} / \mathrm{l}$ & 0.9 & 0.8 & 0.5 & 0.1 & 1.0 \\
\hline LDL-C & $<4.4 \mathrm{mmol} / \mathrm{l}$ & 2.8 & 2.5 & 2.0 & 0 & 2.9 \\
\hline Triglycerides & $0.6-2.2 \mathrm{mmol} / \mathrm{l}$ & 2.6 & 1.8 & 2.8 & 1.8 & 3.5 \\
\hline $\operatorname{IgA}$ & $0.70-4.00 \mathrm{~g} / 1$ & & & & 0.22 & 0.30 \\
\hline $\mathrm{IgG}$ & $7.0-16.0 \mathrm{~g} / 1$ & & & & 60.3 & 6.9 \\
\hline $\operatorname{IgM}$ & $0.40-2.30 \mathrm{~g} / 1$ & & & & 0.07 & 0.24 \\
\hline M-protein & Absent $(\mathrm{g} / \mathrm{l})$ & & & & 41.7 & 3.7 \\
\hline Total protein & $60-80 \mathrm{~g} / 1$ & & & & 111 & 57 \\
\hline
\end{tabular}

Both total and HDL-cholesterol concentrations decrease over the years until very low HDL-C levels were measured. After correction of the paraproteinemia with successive therapy of melfalan-prednisolon and later bortezomib-dexamethason, cholesterol levels restored to initial values

colorimetrically detected and used as a measure for the amount of HDL-C [16]. Interference of paraproteins by precipitate formation in the first step gives falsely increased absorbance of the blank and falsely decreased HDL-C values after subtraction from the final absorbance. This interference is unpredictable and independent of the 
quantity or isotype of paraprotein [10-14]. In the conventional precipitation method for measuring HDL-C, $\mathrm{MgCl}_{2}$ and sodium phosphotungstate are manually added reagents to serum. They cause low density lipoproteins like chylomicrons, VLDL and LDL to precipitate and separate from high density lipoproteins, which remain in the supernatant. Total cholesterol is measured in the supernatant as a measure for the amount of HDL-C. This manual one-step precipitation assay is not interfered by paraproteins. The difference in HDL-C between the homogenous assay $(0.1 \mathrm{mmol} / \mathrm{l})$ and the manual precipitation assay $(1.2 \mathrm{mmol} / \mathrm{l})$ is therefore most probably due to the presence of paraproteins. This suggestion is supported by the improvement of HDL-C $(1.0 \mathrm{mmol} / \mathrm{l})$ after correction of the paraproteinemia in response to therapy.

Thirdly, according to some reports, measuring (almost) undetectable HDL-C values is extremely rare. In 2002, only $16(0.06 \%)$ out of 247.111 HDL-C measurements in the Clinical Laboratory of the San Francisco Medical Center (University of California) yielded undetectable values [11]; paraprotein interference associated with undetectable HDL-C values was identified in 3 (16\%) of 19 paraproteincontaining specimens. In another small series of patients with known monoclonal paraproteins, an extremely low or undetectable HDL-C value was found in 4 out of 13 patients [12]. Recently, Roche Diagnostics has replaced the second generation HDL-C test by a new third generation test with an improved start reagent (R1). Although the frequency of the interference is further reduced we have observed few other cases with falsely low HDL-C caused by paraprotein interference since this replacement.

Fourthly, because of the anemia (and extremely high ESR) on the first day of acute illness, an underlying chronic condition could have been considered earlier. Would a more earlier recognition of the underlying multiple myeloma have benefitted the patient? The extremely low HDL-C values were measured 3 months before the first hospital admittance by the general practitioner. The initial treatment of the patient would not have been different, but a recurrence of the pneumonia might have been postponed by an earlier treatment of the multiple myeloma.

Thus, when measuring extremely low HDL-C levels on routine cardiovascular screening, cardiologists and other physicians may consider the presence of multiple myeloma as a relatively rare but clinically important differential diagnosis.

Conflict of interest statement No conflict of interest reported.

Open Access This article is distributed under the terms of the Creative Commons Attribution Noncommercial License which permits any noncommercial use, distribution, and reproduction in any medium, provided the original author(s) and source are credited.

\section{References}

1. Anderson KM, Castelli WP, Levy D (1987) Cholesterol and mortality: 30 years of follow-up from the Framingham study. JAMA 257:2176-2180

2. Assmann G, Gotto AM (2004) HDL cholesterol and protective factors in atherosclerosis. Circulation 109:III-8-III-14

3. Shor R, Wainstein J, Oz D et al (2008) Low HDL levels and the risk of death, sepsis and malignancy. Clin Res Cardiol 97:227233

4. Murali MR, Kratz A, Finberg KE (2006) Case 40-2006: a 64-year-old man with anemia and a low level of HDL cholesterol. NEJM 355:2772-2779

5. Schifferli JA (2007) Case 40-2006: anemia and low HDL cholesterol. NEJM 356:1893-1895

6. Jung C, Fischer N, Fritzenwanger M et al (2008) Endothelial progenitor cells in adolescents: impact of overweight, age, smoking, sport and cytokines in younger age. Clin Res Cardiol 98:179-188

7. Schunkert H, Moebus S, Hanisch J et al (2008) The correlation between waist circumference and ESC cardiovascular risk score: data from the German metabolic and cardiovascular risk project (GEMCAS). Clin Res Cardiol 97:827-835

8. Greipp PR, San Miguel J, Durie BG et al (2005) International staging system for multiple myeloma. J Clin Oncol 23:34123420

9. International Myeloma Working Group (2003) Criteria for the classification of monoclonal gammopathies, multiple myeloma and related disorders: a report of the International Myeloma Working Group. Br J Haematol 121:749-757

10. Smogorzewska A, Flood JG, Long WH et al (2004) Paraprotein interference in automated chemistry analyzers. Clin Chem 50:1691-1693

11. Baca A, Haber RJ, Sujishi K et al (2004) Artifactual undetectable HDL-cholesterol with the Beckman Synchron LX and Vitros 950 Assays temporally associated with a paraprotein. Clin Chem 50:255-256

12. Kadri N, Douville P, Lachance P (2002) Monoclonal paraprotein may interfere with the Roche Direct HDL-C Plus assay. Clin Chem 48:964

13. Yang Y, Howanitz PJ, Howanitz JH et al (2008) Paraproteins are a common cause of interferences with automated chemistry methods. Arch Pathol Lab Med 132:217-223

14. Tsai LY, Tsai SM, Lee SC et al (2005) Falsely low LDL-cholesterol concentrations and artifactual undetectable HDL-cholesterol measured by direct methods in a patient with monoclonal paraprotein. Clin Chim Acta 358:192-195

15. Merlini G, Farhangi M, Osserman EF (1986) Monoclonal immunoglobulins with antibody activity in myeloma, macroglobulinemia and related plasma cell dyscrasias. Semin Oncol 13:350-365

16. Warnick GR, Nauck M, Rifai N (2001) Evolution of methods for measurement of HDL-cholesterol: from ultracentrifugation to homogeneous assays. Clin Chem 47:1579-1596 